Article

\title{
Hirota Difference Equation and Darboux System: Mutual Symmetry
}

\author{
Andrei Pogrebkov 1,2, + iD \\ 1 Steklov Mathematical Institute, Moscow 119991, Russia; pogreb@mi-ras.ru; Tel.: +7-495-984-81-41 \\ 2 Department of Mathematics, National Research University Higher School of Economics, \\ Moscow 119048, Russia \\ † Current address: Gubkin str. 8, Steklov Mathematical Institute, Moscow 119991, Russia.
}

Received: 1 March 2019; Accepted: 21 March 2019; Published: 25 March 2019

\begin{abstract}
We considered the relation between two famous integrable equations: The Hirota difference equation (HDE) and the Darboux system that describes conjugate curvilinear systems of coordinates in $\mathbb{R}^{3}$. We demonstrated that specific properties of solutions of the HDE with respect to independent variables enabled introduction of an infinite set of discrete symmetries. We showed that degeneracy of the HDE with respect to parameters of these discrete symmetries led to the introduction of continuous symmetries by means of a specific limiting procedure. This enabled consideration of these symmetries on equal terms with the original HDE independent variables. In particular, the Darboux system appeared as an integrable equation where continuous symmetries of the HDE served as independent variables. We considered some cases of intermediate choice of independent variables, as well as the relation of these results with direct and inverse problems.
\end{abstract}

Keywords: Hirota difference equation; Darboux system; integrable equations; symmetries; compatibility; inverse problem

\section{Introduction}

The Hirota bilinear difference equation (HBDE) was introduced in References [1,2] and has received a lot of attention in the literature, e.g., References [3-15], because since References [2,3] this equation has been known to generate many discrete and continuous integrable equations, such as the Kadomtsev-Petviashvili equation (KP), modified Kadomtsev-Petviashvili equation, two-dimensional Toda lattice, sine-Gordon equation, Benjamin-Ono equation, etc. This equation is often considered to be a fundamental integrable system, while it appears also in the study of quantum transfer matrices. Detailed reviews of the results related to this equation are given in References [6,7] (see also References therein). Following Reference [7], we write this equation in "potential" form as an equation on a real function $v(m)=v\left(m_{1}, m_{2}, m_{3}\right)$ of three discrete variables (numbers) $m_{i} \in \mathbb{Z}$ :

$$
v^{(1,2)}\left(v^{(2)}-v^{(1)}\right)+v^{(2,3)}\left(v^{(3)}-v^{(2)}\right)+v^{(3,1)}\left(v^{(1)}-v^{(3)}\right)=0 .
$$

Hereafter, we denote by upper indexes 1,2, and 3 in parenthesis shifts of independent variables:

$$
\begin{gathered}
v^{(1)}(m)=v\left(m_{1}+1, m_{2}, m_{3}\right), \quad v^{(2)}(m)=v\left(m_{1}, m_{2}+1, m_{3}\right), \text { etc., } \\
v^{(i, j)}=\left(v^{(i)}\right)^{(j)} \equiv\left(v^{(j)}\right)^{(i)} \text { for any } i, j=1,2,3 .
\end{gathered}
$$


Equation (1) has Lax representation (e.g., see Reference [6]) with the Lax pair, which is given by any two of the following three equations:

$$
\begin{aligned}
& \varphi^{(2)}(m, \lambda)=\varphi^{(1)}(m, \lambda)+\left(v^{(2)}(m)-v^{(1)}(m)\right) \varphi(m, \lambda), \\
& \varphi^{(3)}(m, \lambda)=\varphi^{(2)}(m, \lambda)+\left(v^{(3)}(m)-v^{(2)}(m)\right) \varphi(m, \lambda), \\
& \varphi^{(1)}(m, \lambda)=\varphi^{(3)}(m, \lambda)+\left(v^{(1)}(m)-v^{(3)}(m)\right) \varphi(m, \lambda),
\end{aligned}
$$

where $\lambda \in \mathbb{C}$ is a spectral parameter. The sum of these equations is equal to zero identically, nevertheless, we write them here just to emphasize symmetry of the Hirota difference equation (HDE) with respect to all independent variables $m_{i}$.

In Reference [12], we considered direct and inverse problems for the HDE Equation (1) in a class of solutions $v(m)$ growing linearly at infinity. More exactly, let $a_{1}, a_{2}$ and $a_{3}$ be real constants, such that:

$$
a_{i} \neq a_{j}, \text { for } i \neq j \text {. }
$$

Then we put:

$$
v(m)=u(m)-a_{1} m_{1}-a_{2} m_{2}-a_{3} m_{3},
$$

where $u(m)$ tends to a constant rapidly enough if some $m_{i} \rightarrow \infty$. In particular, this means that:

$$
v^{(j)}-v^{(i)} \rightarrow a_{i j} \text {, where } a_{i j}=a_{i}-a_{j} .
$$

Strictly speaking, the Lax equations should be substituted by:

$$
\varphi^{(i)}=\varphi^{(j)}+\left(u^{(i)}-u^{(j)}+a_{j i}\right) \varphi, \quad i, j=1,2,3, \quad i \neq j,
$$

but for brevity, we used them in the form of Equations (3)-(5), assuming that $v(m)$ obeys Equation (8). The same must be taken into account for the HDE in Equation (1). Notice that in the case $u(m) \equiv 0$, the $v(m)$ given by Equation (7) obeys the HDE identically.

The Jost solution $\varphi(m, \lambda)$ of Equations (3)-(5) is defined (see, e.g., Reference [12]) as:

$$
\varphi(m, \lambda)=E(m, \lambda) \chi(m, \lambda), \quad \lambda \in \mathbb{C},
$$

where:

$$
E(m, \lambda)=\left(\lambda-a_{1}\right)^{m_{1}}\left(\lambda-a_{2}\right)^{m_{2}}\left(\lambda-a_{3}\right)^{m_{3}},
$$

and is fixed by normalization condition:

$$
\lim _{\lambda \rightarrow \infty} \chi(m, \lambda)=1
$$

In Reference [12], we proved that function $\chi(m, \lambda)$ obeys $\bar{\partial}$-equation:

$$
\frac{\partial \chi(m, \lambda)}{\partial \bar{\lambda}}=R(m, \lambda) \chi(m, \bar{\lambda})
$$

Here, we denoted:

$$
R(m, \lambda)=\frac{E(m, \bar{\lambda})}{E(m, \lambda)} r(\lambda), \quad \lambda \in \mathbb{C},
$$

where $E(m, \lambda)$ is defined above and $r(\lambda)$ is a function of $\lambda$ only-the spectral data. We assume that the inverse problem (Equation (13)) with the normalization (Equation (12)) defines its solution $\chi(m, \lambda)$ uniquely in the whole range of its variables, at least under some small norm assumptions on $r(\lambda)$. Then, solution $v(m)$ of the HDE is given by Equation (7), where: 


$$
u(m)=\lim _{\lambda \rightarrow \infty} \lambda(\chi(m, \lambda)-1)
$$

Here, we considered the relation of the HDE with another well-known system-the Darboux system that describes the conjugate curvilinear systems of coordinates in three dimensional space with diagonal metrics $g_{i j}$. Investigation of the latter system is a classical problem of differential geometry [16-20]. This system is given by the following six equations on coefficients $\Gamma_{i j}$ :

$$
\partial_{i} \Gamma_{j k}=\partial_{k} \Gamma_{j i}=\Gamma_{j k} \Gamma_{k i}+\Gamma_{j i} \Gamma_{i k}-\Gamma_{j k} \Gamma_{j i}
$$

where $i, j, k=$ perm1,2,3. Coefficients $\Gamma_{i j}$ and $\Gamma_{j i}$ here are just simplified notations for the Christoffel symbols $\Gamma_{k i}^{j}$ for $j=k$ corresponding to diagonal metrics. For us, it was essential that this system be integrable in the sense that it appears as a condition of compatibility of the following three equations:

$$
\frac{\partial^{2} \varphi}{\partial t_{i} \partial t_{j}}=\Gamma_{i j} \frac{\partial \varphi}{\partial t_{i}}+\Gamma_{j i} \frac{\partial \varphi}{\partial t_{j}} \quad i \neq j
$$

(of any two of them, as in the HDE case), linear with respect to function $\varphi\left(t_{1}, t_{2}, t_{3}\right), t \in \mathbb{R}^{3}$. This overdetermined system of equations occurs not only in the differential geometry in $\mathbb{R}^{3}$, but also in description of Hamiltonian systems of hydrodynamic type [21]. In the literature, it was called the Darboux-Zakharov-Manakov system [4,22,23].

Following Reference [21], we introduced three functions $v^{(i)}\left(t_{1}, t_{2}, t_{3}\right), i=1,2,3$, such that:

$$
\Gamma_{i j}=\frac{v_{t_{j}}^{(i)}}{v^{(j)}-v^{(i)}}
$$

where now the upper index just distinguishes these functions, and subscript denotes derivative with respect to $t_{j}$. The system of Equation (16) with respect to $\Gamma_{i j}$ is equivalent to the following system of equations on $v^{(i)}$ :

$$
v_{t_{j}, t_{k}}^{(i)}=v_{t_{j}}^{(i)} \frac{v_{t_{k}}^{(i)}-v_{t_{k}}^{(j)}}{v^{(i)}-v^{(j)}}+v_{t_{k}}^{(i)} \frac{v_{t_{j}}^{(k)}-v_{t_{j}}^{(i)}}{v^{(k)}-v^{(i)}}+\frac{v_{t_{k}}^{(i)} v_{t_{j}}^{(k)}-v_{t_{j}}^{(i)} v_{t_{k}}^{(j)}}{v^{(j)}-v^{(k)}}
$$

where $i \neq j \neq k \neq i$. In these terms, the Lax pair is given by any two of the following three equations:

$$
\begin{aligned}
& \left(v^{(1)}-v^{(2)}\right) \varphi_{t_{1} t_{2}}+v_{t_{2}}^{(1)} \varphi_{t_{1}}-v_{t_{1}}^{(2)} \varphi_{t_{2}}=0, \\
& \left(v^{(2)}-v^{(3)}\right) \varphi_{t_{2} t_{3}}+v_{t_{3}}^{(2)} \varphi_{t_{2}}-v_{t_{2}}^{(3)} \varphi_{t_{3}}=0, \\
& \left(v^{(3)}-v^{(1)}\right) \varphi_{t_{3} t_{1}}+v_{t_{1}}^{(3)} \varphi_{t_{3}}-v_{t_{3}}^{(1)} \varphi_{t_{1}}=0,
\end{aligned}
$$

and Equation (19) is the compatibility condition of these equations.

The article is organized as follows: In Section 2.1, we demonstrate that specific properties of solutions of the HDE with respect to independent variables enable introduction of an infinite set of discrete symmetries. We also prove that degeneracy of the HDE with respect to parameters of these discrete symmetries leads to introduction of a set of continuous symmetries of the HDE. We prove mutual compatibility of these symmetries, as well as their compatibility with the original HDE evolution. This enables us to consider continuous parameters of these symmetries on equal terms with the discrete independent variables of the HDE. The corresponding integrable equations, including the Darboux system, are constructed by these means in Section 2.2. In Section 2.3, we describe the relation of the introduced symmetries with the inverse problem. In particular, we prove that solutions of the Darboux system include a subclass of solutions that obeys the HDE, as well. Concluding remarks and possible ways to generalize our results are given in Section 3. 


\section{Results}

\subsection{From Ill-Posedness of the HDE to Its Symmetries}

Equation (1) can be considered naturally as an evolution equation, where, say, $m_{1}$ and $m_{2}$ play the role of space variables, and $m_{3}$ is the time one, i.e., one can consider the Cauchy problem:

$$
v\left(m_{1}, m_{2}, 0\right)=v_{0}\left(m_{1}, m_{2}\right)
$$

where $v_{0}$ is some given function. But it is easy to check directly that this problem has two trivial solutions: $v(m)=v_{0}\left(m_{1}+m_{3}, m_{2}\right)$ and $v(m)=v_{0}\left(m_{1}, m_{2}+m_{3}\right)$, i.e., solutions that obey $v^{(3)} \equiv v^{(1)}$ or $v^{(3)} \equiv v^{(2)}$. Thus the initial problem (23) for Equation (1) is ill-posed. In References [12,13], we resolved this ill-definiteness by assuming the linear growth of the solution at infinity. More exactly, we imposed on $v$ asymptotic behavior in Equations (6)-(8), which excludes the cases $v^{(i)} \equiv v^{(j)}$. In Reference [12], we proved by means of the inverse scattering transform that this condition is also sufficient for solvability of the above mentioned Cauchy problem in the class of rapidly decaying $u(m)$, as in Equation (7).

Formulation of the inverse problem in Equations (13) and (14) demonstrates a specific property of the Hirota difference equation: for an arbitrary set of pairwise different real constants $a_{i}, i=1,2, \ldots$, one can introduce a corresponding number of independent variables $m_{i}, i=1,2, \ldots$, in a way that with respect to any three different variables function $v(m)=v\left(m_{1}, m_{2}, \ldots\right)$ obeys the same Equation (1) (see also [11]). Here, we prove this directly by means of the Lax representation (Equations (3)-(5)). In order to explicitly specify the set of independent variables involved in the Lax operators and the HDE, we use notation $L(i, j)$ for the equation as:

$$
L(i, j): \quad \varphi^{(i)}-\varphi^{(j)}=\left(v^{(i)}-v^{(j)}\right) \varphi, \quad i \neq j .
$$

Compatibility of the pair of these equations, $L(i, j)$ and $L(i, k)$, with one common index is equivalent to the HDE (Equation (1)):

$$
v^{(i, j)}\left(v^{(j)}-v^{(i)}\right)+v^{(j, k)}\left(v^{(k)}-v^{(j)}\right)+v^{(k, i)}\left(v^{(i)}-v^{(k)}\right)=0, \quad i \neq j \neq k,
$$

with respect to the independent variables $m_{i}, m_{j}$, and $m_{k}$, so we denote this equation by $H(i, j, k)$. Summing up the left-hand (and right-hand) sides of equalities (Equation (24)), we have identity:

$$
L(i, j)+L(j, k)+L(k, i) \equiv 0
$$

that is valid independently of the validity of these equalities themselves. Thus, every $\operatorname{HDE} H(i, j, k)$ is condition of compatibility of any two equations of the three: $L(i, j), L(j, k)$, and $L(k, i)$, and the third one is compatible, as well.

For a given $H(i, j, k)$ evolution with respect to any other $m_{l}$, where $l \neq i, j, k$, is nothing but a discrete symmetry. Action of this symmetry on the dependent variable $v\left(m_{i}, m_{j}, m_{k}\right)$ is given by means of the corresponding equations $L(l, i), L(l, j)$, and $L(l, k)$ (see Equation (24)). These equations must be mutually compatible and compatible with equations $L(i, j), L(j, k)$, and $L(k, i)$, that generates $H(i, j, k)$ itself. As we mentioned above, compatibility of equations $L(i, j)$ and $L(j, l)$ gives equation $H(l, i, j)$, and the same is valid for all other cases with one common index in operators $L$. Thus, we get that also equations $H(l, j, k)$, and $H(l, k, i)$ are satisfied. Next, we have to consider compatibility of the pair, say, $L(i, j)$ and $L(k, l)$, where all indexes are different. This results in equality:

$$
v^{(j, k)}\left(v^{(k)}-v^{(j)}\right)+v^{(k, i)}\left(v^{(i)}-v^{(k)}\right)+v^{(i, l)}\left(v^{(l)}-v^{(i)}\right)+v^{(l, j)}\left(v^{(j)}-v^{(l)}\right)=0,
$$

that looks to be an equation with respect to four independent variables. It is not the case because, thanks to Equation (26), we can write $L(k, l)=L(i, k)+L(l, i)$, so that compatibility of the left hand 
side (l.h.s.) with $L(i, j)$ follows from compatibility of $L(i, j)$ with two equations in the right hand side that is already established. In fact, it is easy to check directly that Equation $(27)=H(i, j, k)+H(j, i, l)$.

Setting that function $v(m)=v\left(m_{1}, m_{2}, m_{3}, \ldots\right)$ depends on an arbitrary number of independent variables, we have to substitute Equation (7) by means of the equality:

$$
v(m)=u(m)-\sum_{i} a_{i} m_{i}
$$

where $u(m) \equiv u\left(m_{1}, m_{2}, m_{3}, \ldots\right) \rightarrow 0$ at $m$-infinity, and where $a_{i}$ are real constants that parametrize linear growth of $v(m)$ at infinity. All these parameters must obey the condition in Equation (6) for any $i \neq j$. As well, $E(m, \lambda)$ in Equation (11) sounds now as:

$$
E(m, \lambda)=\prod_{i}\left(\lambda-a_{i}\right)
$$

Formulation of the inverse problem (Equations (12)-(14)), where $E(m, \lambda)$ is given above, demonstrates that in the limit $a_{i} \rightarrow a_{j}$, the spectral data $R(m, \lambda)$ and then the Jost solution and function $u(m)$ depend on the sum $m_{i}+m_{j}$ only. Thanks to Equation (28) the same is valid for the function $v(m)$ (see Reference [13] for more detail). Thus, in this limit, $v^{(i)}(m)=v^{(j)}(m)$ and corresponding equation $H(i, j, k)$ becomes identity, as we discussed above. In References [14,15], we mentioned that this specific degeneracy of the HDE enables introduction of the continuous symmetries, i.e., symmetries parametrized by the continuous variables. Let us consider limit $a_{2} \rightarrow a_{1}$ as an example. We introduce function $u\left(m_{1}, m_{3}, t_{1}\right)$ by means of the limit procedure:

$$
\begin{gathered}
u_{t_{1}}^{(1)}\left(m_{1}+m_{2}, m_{3}, t_{1}\right)=\lim _{a_{2} \rightarrow a_{1}} \frac{u^{(1)}\left(m_{1}, m_{2}, m_{3}\right)-u^{(2)}\left(m_{1}, m_{2}, m_{3}\right)}{a_{21}} \equiv \\
\equiv \lim _{a_{2} \rightarrow a_{1}} \frac{u\left(m_{1}+1, m_{2}, m_{3}\right)-u\left(m_{1}, m_{2}+1, m_{3}\right)}{a_{21}} .
\end{gathered}
$$

In a generic case, we introduce "time" $t_{j}$ by equality:

$$
u_{t_{j}}^{(j)}=\lim _{a_{i} \rightarrow a_{j}} \frac{u^{(i)}-u^{(j)}}{a_{j i}}
$$

and the same is definition of $t_{j}$-dependence of $v(m, t), \varphi(m, t)$, and $\chi(m, t)$. In other words, in all these cases, we write, say,

$$
v^{(i)}=v^{(j)}+a_{i j} v_{t_{j}}^{(j)}+o\left(a_{i j}\right), \quad a_{i j} \rightarrow 0,
$$

etc., and consider the first order terms with respect to $a_{i j}$. Thus, thanks to Equation (28), we have that:

$$
v_{t_{i}}^{(i)}=1+u_{t_{i}}^{(i)}
$$

and thanks to Equation (10):

$$
\varphi_{t_{i}}^{(i)}(m, t, \lambda)=E^{(i)}(m, \lambda)\left(\frac{\chi^{(i)}(m, t, \lambda)}{\lambda-a_{i}}+\chi_{t_{i}}^{(i)}(m, t, \lambda)\right)
$$

Taking that the $t$-dependence appears as the limit procedure for discrete symmetries into account, it is natural to expect that it gives continuous symmetries of the HDE. To show this explicitly, we have to consider corresponding limits of Equations (24) and (25). It is clear that $L(i, k) \rightarrow L(j, k)$ if $a_{i} \rightarrow a_{j}$, $k \neq j$, as well as $H(i, k, l) \rightarrow H(j, k, l)$ for any $k, l \neq j$. Taking that $L(i, j)$ and $H(i, j, k)$ become identities in this limit, we define: 


$$
L(j \mid j)=\lim _{a_{i} \rightarrow a_{j}} L(j, i) / a_{i j}, \quad H(j, k \mid j)=\lim _{a_{i} \rightarrow a_{j}} H(j, k, i) / a_{i j}
$$

so that by Equations (24) and (28) we get:

$$
L(i \mid i): \quad \varphi_{t_{i}}^{(i)}=v_{t_{i}}^{(i)} \varphi .
$$

Next, in the first order of $a_{k i}$ equation $H(i, j, k)$ (see Equation (25)) reduces to:

$$
H(i, j \mid i): \quad v_{t_{i}}^{(i, j)}\left(v^{(j)}-v^{(i)}\right)=v_{t_{i}}^{(i)}\left(v^{(j)}-v^{(i)}\right)^{(i)}, \quad i \neq j,
$$

which is exactly the condition of compatibility of the equation $L(i \mid i)$ with equations $L(i, j), j \neq i$.

Similarly, let us start with the set of six discrete variables, say, $\left\{m_{1}, \ldots, m_{6}\right\}$ under the condition that all $H(i, j, k), i, j, k=1, \ldots, 6$, are valid. Then we can consider limits $a_{i+3} \rightarrow a_{i}, i=1,2,3$. Denoting corresponding "times" (continuous parameters) as $t_{1}, t_{2}$, and $t_{3}$, we derive three equations $L(i \mid i), i=1,2,3$, (see Equation (35)) that guarantee that dependence on these parameters gives symmetries of the $H(1,2,3)$. On the other side, these symmetries must be mutually compatible, i.e., equations $L(i \mid i)$ and $L(j \mid j)$ must be compatible for all $i \neq j$ if $L(i, j)$ is fulfilled. Thanks to Equation (31) this gives:

$$
\left(v_{t_{i}}^{(i)} v\right)_{t_{j}}^{(j)}=\left(v_{t_{j}}^{(j)} v\right)_{t_{i}}^{(i)}, \quad i, j=1,2,3
$$

It is necessary to mention that Equation (27) in the first order of $a_{i j}$ under substitution (Equation (31)) reduces to:

$$
\left(v_{t_{i}}^{(i)} v\right)^{(k)}-\left(v_{t_{i}}^{(i)} v\right)^{(l)}=\left(\left(v^{(k)}-v^{(l)}\right) v\right)_{t_{i}}^{(i)}
$$

that like Equation (27) can be written as $H(i k \mid i)-H(i, l \mid i)$ by means of notation (Equation (34)) (cf. Equation (36)). Next, setting in analogy to Equation (30) $v^{(l)}=v^{(k)}+a_{k l} v_{t_{k}}^{(k)}+o\left(a_{k l}\right)$, we again derive Equation (37) (up to change $j \leftrightarrow k$ ) in the first order of Equation (38) with respect to $a_{k l}$. Notice that Equation (37) involves four independent variables: $m_{i}, m_{j}, t_{i}$, and $t_{j}$ similarly to Equations (27) and (38), but in contrast, it is unclear if Equation (37) can be written as combination of two three-dimensional equations.

\subsection{Symmetries and Integrable Equations}

Existence of the symmetries introduced above and their mutual compatibility demonstrates that we can consider them equally with the independent variables of the HDE. To be more exact, let now $u$ and $\varphi$ depend on six independent variables: Three discrete variables $m_{i}$ and three continuous ones $t_{i}$, $i=1,2,3$.

We know that choosing discrete variables $m_{1}, m_{2}$, and $m_{3}$ as independent ones, we get the Hirota difference equation $H(1,2,3)$, i.e., Equation (1). In this case variables $t_{i}, i=1,2,3$ are parameters of the continuous symmetries. Now we choose two discrete and one continuous variables to be independent ones, say, $m_{1}, m_{2}$, and $t_{1}$. Zero curvature condition of these three variables is given by compatibility of the equations $L(1,2)$ and $L(1 \mid 1)$, so it is given by equation $H(1,2 \mid 1)$ in Equation (36). In this case, variables $m_{3}, t_{1}$, and $t_{2}$ play the role of symmetries of this equation, correspondingly the discrete and continuous ones. Next, following Reference [15], we choose one discrete and two continuous variables: $m_{1}, t_{1}$, and $t_{2}$. This choice of variables determines the choice of $L(1 \mid 1)$ in Equation (35) as the first operator of the Lax pair. But, in this case, we can use neither $L(1,2)$ nor $L(2 \mid 2)$ as the second operator of the Lax pair in contrast to the above: We have no shift with respect to the second discrete variable. This shift of the Jost solution can be excluded from $L(2 \mid 2)$ by means of the derivative of the equation $L(1,2)$ (see Equation $(24)$ ), with respect to $t_{2}$. Thus, we get the second equation of the Lax pair in the form: 


$$
\varphi_{t_{2}}^{(1)}=w \varphi_{t_{2}}+v_{t_{2}}^{(1)} \varphi,
$$

where we denoted $w=v^{(1)}-v^{(2)}$. In terms of this evolution, $v^{(1)}$ denotes the shift $m_{1} \rightarrow m_{1}+1$ in the argument of the function $v(m, t)$ in correspondence to Equation (2a), while the upper index of $v^{(2)}$ now denotes only a function, different from $v$. Compatibility condition now sounds as:

$$
\begin{aligned}
& \partial_{t_{2}} \log v_{t_{1}}=\frac{v_{t_{2}}}{w^{(-1)}}-\left(\frac{v_{t_{2}}}{w^{(-1)}}\right)^{(1)}, \\
& \partial_{t_{1}} \log w=\left(\frac{v_{t_{1}}}{w^{(-1)}}\right)^{(1)}-\frac{v_{t_{1}}}{w^{(-1)}},
\end{aligned}
$$

where the first equation can be considered as the evolution one on the function $v(m, t)$, while the second stands as definition of an auxiliary function $w(m, t)$. Complimentary variables $m_{2}, m_{3}$, and $t_{3}$ are parameters of the symmetries of this system.

Finally, we consider the case where all three continuous variables are chosen to be independent variables of some differential equation. In this case, neither equations $L(i, j)$ nor $L(i \mid i)$ can be used in the Lax pair: Shifts of the function $\varphi(m, \lambda)$ with respect to the discrete variables are not allowed. So, we have to use the same trick as above: To exclude these shifts, we differentiate $L(i \mid i)$, as in Equation (35), with respect to $t_{j}, j \neq i$, that gives $\varphi_{t_{i} t_{j}}^{(i)}=v_{t_{i} t_{j}}^{(i)} \varphi+v_{t_{i}}^{(i)} \varphi_{t_{j}}$. Then the compatibility, i.e., the zero curvature condition reads as:

$$
\left(\varphi^{(i)}-\varphi^{(j)}\right) t_{i} t_{j}=\left(v^{(i)}-v^{(j)}\right) t_{t_{i} t_{j}} \varphi+v_{t_{i}}^{(i)} \varphi_{t_{j}}-v_{t_{j}}^{(j)} \varphi_{t_{i}}, \quad i \neq j .
$$

Substituting difference in the 1.h.s. by means of Equation (24), we derive exactly three equations, Equations (20)-(22), for different choices of $i, j=1,2,3$. In other words, we get the Lax representation for the Darboux system. Now discrete variables are parameters of the discrete symmetries, i.e., Darboux transformations of the Darboux system, while from the point of view of the latter equations functions $v^{(i)}, i=1,2,3$ are just different functions that are not obliged to be related by any transformation. In the next section, we briefly consider relation of this approach with the inverse scattering transform.

\subsection{Symmetries and the Inverse Scattering Transform}

The above consideration proves that the equations HDE in Equation (1), $H(i, j \mid k)$ in Equation (36), system Equations (40) and (41), and the Darboux system (Equation (19)) have in common solution $v(m, t)$ depending on all six variables $m_{i}, t_{i}, i=1,2,3$ and evolutions with respect to any pair of these variables are compatible. But this does not mean that any solution of one of these equations with respect to the corresponding three variables admits compatible introduction of other three variables, in the way that $v(m, t)$ obeys other equations of the list. To clarify this point, we briefly consider the relation of these symmetries with the corresponding scattering problems. To proceed, notice that because of Equation (33), we have to modify relations (Equations (10) and (11)) as:

$$
\begin{aligned}
\varphi(m, t, \lambda) & =E(m, t, \lambda) \chi(m, t, \lambda), \\
E(m, t, \lambda) & =\left(\lambda-a_{1}\right)^{m_{1}}\left(\lambda-a_{2}\right)^{m_{2}}\left(\lambda-a_{3}\right)^{m_{3}} \times \\
& \times \exp \left(\frac{t_{1}}{\lambda-a_{1}}+\frac{t_{2}}{\lambda-a_{2}}+\frac{t_{3}}{\lambda-a_{3}}\right),
\end{aligned}
$$

and to impose condition that asymptotically:

$$
\chi(m, t, \lambda) \rightarrow 1 \text {, when } m \rightarrow \infty \text {, or } t \rightarrow \infty,
$$

so that Equations (24) and (35) take the form: 


$$
\begin{aligned}
& \left(\lambda-a_{i}\right) \chi^{(i)}(m, t, \lambda)=\left(\lambda-a_{j}\right) \chi^{(j)}(m, t, \lambda)+\left(v^{(i)}-v^{(j)}\right) \chi(m, t, \lambda), \quad i \neq j, \\
& \left(\lambda-a_{i}\right) \chi_{t_{i}}^{(i)}(m, t, \lambda)+\chi^{(i)}(m, t, \lambda)=v_{t_{i}}^{(i)}(m, t) \chi(m, t, \lambda) .
\end{aligned}
$$

Asymptotically, see Equations (8) and (32), where we have that:

$$
v^{(i)}(t)-v^{(j)}(t) \rightarrow a_{j i} \neq 0, \quad v_{t_{j}}^{(i)}(t) \rightarrow 1 .
$$

The inverse problem for the HDE, given by Equations (12)-(14) admits switching on dependence on continuous variables $t_{i}$ by replacing $R(m, \lambda)$ in Equation (13) with:

$$
R(m, t, \lambda)=\frac{E(m, t, \bar{\lambda})}{E(m, t, \lambda)} r(\lambda)
$$

where $E(m, t, \lambda)$ is defined in Equation (43). The same is valid for the equation $H(i, j \mid k)$ in Equation (36), because its linear problem is given by the same equation $L(i, j)$ as the linear problem of HDE, i.e., the inverse problem is also given by Equations (12) and (13) with the above substitution. But situation changes, if we consider the system equation (Equations (40) and (41)), where the linear problem (i.e., the Lax operator) is given by equation $L(1 \mid 1)$ in Equation (35), i.e., Equation (46) with $i=1$ in terms of $\chi$. Taking the asymptotic behavior in Equation (47) into account, we consider function $u(t, m)$ in Equation (32) as perturbation. So the "bare" equation, i.e., equation on $\chi$ that corresponds to $u(m, t) \equiv 0$, sounds as:

$$
\left(\lambda-a_{1}\right) \chi_{t_{1}}^{(1)}(m, t, \lambda)+\chi^{(1)}(m, t, \lambda)-\chi(m, t, \lambda)=0 .
$$

Let function $g(m, t, \lambda)$ be the Green's function of this equation:

$$
\left(\lambda-a_{1}\right) g_{t_{1}}^{(1)}\left(m_{1}, t_{1}, \lambda\right)+g^{(1)}\left(m_{1}, t_{1}, \lambda\right)-g\left(m_{1}, t_{1}, \lambda\right)=\delta_{m_{1}, 0} \delta\left(t_{1}\right) .
$$

Then solution of Equation (46) with the normalization of Equation (44) is given by integral equation:

$$
\chi\left(m_{1}, t_{1}, \lambda\right)=1+\int d t_{1}^{\prime} \sum_{n_{1}} g\left(m_{1}-n_{1}, t_{1}-t_{1}^{\prime}, \lambda\right) u_{t_{1}}^{(1)}\left(m_{1}^{\prime}, t_{1}^{\prime}\right) \chi\left(m_{1}^{\prime}, t_{1}^{\prime}, \lambda\right) .
$$

It is easy to see that the Green's function is given by means of:

$$
g\left(m_{1}, t_{1}, \lambda\right)=\frac{i}{(2 \pi)^{2}} \oint_{|\zeta|=1} d \zeta \int d p_{1} \frac{e^{i p_{1} t_{1}}}{\zeta-1-i p_{1}\left(\lambda-a_{1}\right)},
$$

that has the only departure from analyticity in the $\lambda$-plane, given by continuous (with exception to the point $\left.\lambda=a_{1}\right) \bar{\partial}$-derivative:

$$
\frac{\partial g\left(m_{1}, t_{1}, \lambda\right)}{\partial \bar{\lambda}}=\left(\frac{\bar{\lambda}-a_{1}}{\lambda-a_{1}}\right)^{m_{1}} \exp \left(\frac{t_{1}}{\bar{\lambda}-a_{1}}-\frac{t_{1}}{\lambda-a_{1}}\right) g(\lambda)
$$

where $g(\lambda)$ is a function, explicit form of which is not relevant here. This property of the Green's function assumes that solution of the integral Equation (51) also obeys the continuous (with the same exception) $\bar{\partial}$-derivative. So, its inverse problem is also given by Equations (12) and (13) and admits introduction of other discrete and continuous variables by means of Equation (48).

Consideration of the Darboux problem is more involved. Choosing Equation (3) as the first equation of the Lax pair, we rewrite it, thanks to Equation (42) as equation on $\chi$ : 


$$
\begin{aligned}
& \left(\lambda-a_{1}\right)\left(\lambda-a_{2}\right)\left(v^{(1)}-v^{(2)}\right) \chi_{t_{1}, t_{2}}(t, \lambda)+ \\
& \quad+\left(\lambda-a_{1}\right)\left(\left(\lambda-a_{2}\right) v_{t_{2}}^{(1)}+v^{(1)}-v^{(2)}\right) \chi_{t_{1}}(t, \lambda)- \\
& \quad-\left(\lambda-a_{2}\right)\left(\left(\lambda-a_{1}\right) v_{t_{1}}^{(2)}+v^{(2)}-v^{(1)}\right) \chi_{t_{2}}(t, \lambda)+ \\
& \quad+\left(\left(\lambda-a_{2}\right) v_{t_{2}}^{(1)}-\left(\lambda-a_{1}\right) v_{t_{1}}^{(2)}+v^{(1)}-v^{(2)}\right) \chi(t, \lambda)=0 .
\end{aligned}
$$

Because of asymptotic behavior in Equation (47), the constant (corresponding to $u(m) \equiv 0$ ) part of the operator equals:

$$
L_{0}=\partial_{t_{1}} \partial_{t_{2}}-\frac{\lambda-a_{1}}{a_{12}\left(\lambda-a_{2}\right)} \partial_{t_{1}}+\frac{\lambda-a_{2}}{a_{12}\left(\lambda-a_{1}\right)} \partial_{t_{2}} .
$$

Let us introduce the Green's function:

$$
L_{0} G_{0}(t, \lambda)=\delta(t) \equiv \delta\left(t_{1}\right) \delta\left(t_{2}\right),
$$

where in order to obey Equation (44), we have to construct the Green's function that decays when $t=\left\{t_{1}, t_{2}\right\} \rightarrow \infty$. This condition is satisfied if:

$$
G_{0}(t, \lambda)=\frac{-1}{(2 \pi)^{2}} \int \frac{d^{2} p e^{\left(i p_{1} t_{1}+i p_{2} t_{2}\right) / a_{12}}}{\left(p_{1}-\left(\lambda-a_{2}\right) /\left(\lambda-a_{1}\right)\right)\left(p_{2}-\left(\lambda-a_{1}\right) /\left(\lambda-a_{2}\right)\right)-1} .
$$

It is easy to see that besides the nonzero $\bar{\partial}$-derivative with respect to $\lambda$ in the complex domain, continuous with exception to the points $\lambda=a_{1}$ and $a_{2}$, this function has discontinuity on the circle:

$$
\left|\lambda-\frac{a_{1}+a_{2}}{2}\right|^{2}=a_{12}^{2}
$$

Correspondingly to the above discussion, it is natural to expect that the inverse problem for the function $\chi$ in this case will be different from the one given by Equations (12) and (13): The departure from analyticity of the Green's function (Equation (57)) means that the inverse problem is a combination of the $\bar{\partial}$-problem (Equation (13)) and the nonlocal Riemann-Hilbert problem on the circle (Equation (58)), cf. Reference [24], where the analogous linear problem was considered in detail. To control both these defects of the Jost solution, we need (besides the scattering data $R(m, t, \lambda)$ in Equation (48)) some function $\rho(p, t, \lambda)$, where $p$ is a real parameter and support of this function on the $\lambda$-plane belongs to the circle (Equation (58)).

In summary, let $v^{(i)}\left(t_{1}, t_{2}, t_{3}\right)$ be a solution of the Darboux system parametrized via the inverse problem by means of the two kinds of the scattering data described above. If we want to switch on dependence on discrete variables that are compatible with the original ones, shifts of function $v\left(m_{1}, m_{2}, m_{3}, t_{1}, t_{2}, t_{3}\right)$ give $v^{(i)}$ with respect to Equation (2a), and this function obeys the HDE (Equation (1)) for all $m_{i} \in \mathbb{Z}$, we have to impose the condition that the scattering data $\rho(p, t, \lambda)$ that control discontinuity of the Jost solution on the circle (Equation (58)) vanish.

\section{Discussion}

We presented here a way to derive continuous symmetries of the HDE based on specific degeneration of this equation. Since such degenerations can take place for other difference integrable equations, it is interesting to extend the approach above to these equations. One such equation is the higher HD (see Reference [25]). Another possible generalization of the above approach can be given by the non-Abelian case of the HDE (see Reference [14] and references therein). In this case, there must appear a non-Abelian analog of the Darboux system - an object interesting from both geometric and hydrodynamic points of view.

Finally, it is necessary to mention that the set of solutions of the Darboux system still deserves its detailed description and classification. It is clear that the system admits different classes of solutions 
with very different properties. Some class of solutions of this system was studied in the literature by means of inverse scattering (see Reference [22]). Classes of explicitly solvable solutions of the Darboux system were singled out in Reference [20,26]. These classes are different from the one considered above: Their solutions do not obey the asymptotic condition (Equation (47)). Moreover, generically, these solutions can have singularities. These remarks show that the direct and inverse problems for the Darboux system outlined above deserve much more detailed consideration.

Funding: This work has been partially supported by the Russian Academic Excellence Project ' $5-100$ '.

Acknowledgments: The author thanks R. Ch. Kulaev, M. V. Pavlov, and A. B. Shabat for fruitful discussions.

Conflicts of Interest: The authors declare no conflict of interest.

\section{References}

1. Hirota, R. Nonlinear partial difference equations II; Discrete time Toda equations. J. Phys. Soc. Jpn. 1977, 43, 2074-2078. [CrossRef]

2. Hirota, R. Discrete analogue of a generalized Toda equation. J. Phys. Soc. Jpn. 1981, 50, 3785-3791. [CrossRef]

3. Miwa, T. On Hirota's difference equation. Proc. Jpn. Acad. A Math. Sci. 1982, 58, 9-12. [CrossRef]

4. Zakharov, V.E.; Manakov, S.V. Construction of higher-dimensional nonlinear integrable systems and of their solutions. Funct. Anal. Appl. 1985, 19, 89-101. [CrossRef]

5. Bogdanov, L.V.; Konopelchenko, B.G. Generalized KP hierarchy: Möbius symmetry, symmetry constraints and Calogero-Moser system. Physica D 2001, 152-153, 85-96. [CrossRef]

6. Zabrodin, A.V. Hirota's difference equations. Theor. Math. Phys. 1997, 113, 1347-1392. [CrossRef]

7. Zabrodin, A.V. Bäcklund transformations for the difference Hirota equation and the supersymmetric Bethe ansatz. Theor. Math. Phys. 2008, 155, 567-584. [CrossRef]

8. Saito, S. Octahedral structure of the Hirota-Miwa equation. J. Nonlinear Math. Phys. 2012, 10, 1250032.

9. Krichever, I.; Wiegmann, P.; Zabrodin, A. Elliptic solutions to difference non-linear equations and related many-body problems. Commun. Math. Phys. 1998, 193, 373-396. [CrossRef]

10. Hone, A.N.W.; Kouloukas, T.E.; Ward, C. On Reductions of the Hirota-Miwa Equation. Symmetry Integrab. Geom. Methods Appl. 2017, 13, 057. [CrossRef]

11. Doliwa, A.; Lin, R. Discrete KP equation with self-consistent sources. Phys. Lett. A 2014, 378, 1925-1931. [CrossRef]

12. Pogrebkov, A.K. Hirota difference equation: Inverse scattering transform, Darboux transformation, and solitons. Theor. Math. Phys. 2014, 181, 1585-1598. [CrossRef]

13. Pogrebkov, A.K. Hirota difference equation and a commutator identity on an associative algebra. St. Petersburg Math. J. 2011, 22, 473-483. [CrossRef]

14. Pogrebkov, A.K. Commutator identities on associative algebras. The non-Abelian Hirota difference equation and its reductions. Theor. Math. Phys. 2016, 187, 823-834. [CrossRef]

15. Pogrebkov, A.K. Symmetries of the Hirota difference equation. Symmetry Integrab. Geom. Methods Appl. 2017, 13, 053. [CrossRef]

16. Darboux, G. Lecons sur les Systèmes Orthogonaux et les Coordonnées Curvilignes; Gauthier-Villars: Paris, France, 1898.

17. Eisenhart, L.P. A Treatise on the Differential Geometry of Curves and Surfaces; Kessinger Publishing LLC: Whitefish, MT, USA, 2010.

18. Bianchi, L. Opere. In Sisteme Tripli Orthogonali; Edizioni Cremonese: Roma, Italy, 1956.

19. Rogers, C.; Schief, W.K. BDT, Geometry and Modern Appliation in Soliton Theory; Cambridge University Press: Cambridge, UK, 2002.

20. Ferapontov, E.V. Systems of three differential equations of hydrodynamic type with hexagonal 3-web of characteristics on the solutions. Funct. Anal. Appl. 1989, 23, 151-153. [CrossRef]

21. Tsarev, S.P. The geometry of Hamiltonian systems of hydrodymanic type. The generalized hodograph method. Math. USSR-Izvestiya 1991, 37, 397-419. [CrossRef]

22. Zakharov, V.E. Description of the $n$-orthogonal curvilinear coordinate systems and Hamiltonian integrable systems of hydrodynamic type. I: Integration of the Lamé equations. Duke Math. J. 1998, 94, 103-139. [CrossRef] 
23. Bogdanov L.V.; Konopelchenko, B.G. Generalized integrable hierarchies and Combescure symmetry transformations. J. Phys. A Math. Gen. 1997, 30, 1591-1603. [CrossRef]

24. Garagash, T.I.; Pogrebkov, A.K. Scattering problem for the differential operator $\partial_{x} \partial_{y}+1+a(x, y) \partial_{y}+b(x, y)$. Theor. Math. Phys. 1995, 102, 117-132. [CrossRef]

25. Pogrebkov, A.K. Higher Hirota difference equations and their reductions. Theor. Math. Phys. 2018, 197, 1779-1796. [CrossRef]

26. Kulaev, R.C.; Pogrebkov, A.K.; Shabat, A.B. Darboux system: Liouville reduction and an explicit solution. Proc. Steklov Inst. Math. 2018, 302, 250-269. [CrossRef]

(C) 2019 by the author. Licensee MDPI, Basel, Switzerland. This article is an open access article distributed under the terms and conditions of the Creative Commons Attribution (CC BY) license (http:/ / creativecommons.org/licenses/by/4.0/). 